\title{
Âncoras de decisão: um estudo de precificação com produtos originais e réplicas
}

\author{
Decision anchors: a study on pricing of original products and replicas
}

\author{
Jefferson Rodrigues Pereira \\ Faculdade Novos Horizontes, Programa de Mestrado em Administração, Rua Alvarenga Peixoto, 1270 - 40 andar. CEP.: \\ 30.180-121. Belo Horizonte / Minas Gerais - Brasil, jeffersonrodrigues@live.com
}

\section{Caissa Veloso e Sousa}

Faculdade Novos Horizontes, Programa de Mestrado em Administração, CEP.: 30.180-121. Belo Horizonte / Minas Gerais Brasil, caissa.sousa@unihorizontes.br

\section{Gustavo Rodrigues Cunha}

Faculdade Novos Horizontes, Programa de Mestrado em Administração, CEP.: 30.180-121. Belo Horizonte / Minas Gerais Brasil, gustavo.cunha@unihorizontes.br

\section{Wendel Alex Castro Silva}

Faculdade Novos Horizontes, Programa de Mestrado em Administração, CEP.: 30.180-121. Belo Horizonte / Minas Gerais Brasil,wendel.silva@unihorizontes.br

\section{Eulene Aparecida Machado}

Faculdade Novos Horizontes, Programa de Mestrado em Administração, CEP.: 30.180-121. Belo Horizonte / Minas Gerais Brasil, eulene10@yahoo.com.br

\section{Resumo}

0 presente estudo tem como tema a influência de vieses cognitivos no processo de tomada de decisão do consumidor. Como objetivo geral pretendeu-se identificar a existência de diferenças estatísticamente significantes na precificação de produtos similares, porém de marcas distintas, tendo por base a atribuição de âncoras de decisão. Para tal, utilizou-se uma estrutura metodológica quantitativa, por meio de um estudo de campo do tipo survey online. Finalizaram o questionário 111 entrevistados. Todos os respondentes residem no estado de Minas Gerais, que é o segundo mais populoso do Brasil. Utilizou como método de análise a estatística univariada, calculando-se as médias das diferenças, utilizando para tanto o T-Test de grupos pareados. Em um ambiente de análise de dados foi possível observar diferenças estatisticamente significantes entre as amplitudes das médias. Dentre os principais resultados identificou-se considerável influência de aspectos relacionados à marca nos processos cognitivos de percepção e atribuição de valor a determinados produtos, bem como a presença do viés ancoragem e ajustamento na tomada de decisão do consumidor.

Palavras-chave: Comportamento do consumidor, precificação, tomada de decisão, ancoragem, marcas.

\begin{abstract}
The theme of this study is the influence of cognitive bias on consumer decision-making processes. The overall objective was to identify the existence of statistically significant differences in pricing of similar products, but different brands, when anchors were present. To this end, a quantitative methodological structure was used and a field study was conducted via on-line surveys. 111 respondents completed the questionnaire. All respondents live in the state of Minas Gerais, which is the second most populous in Brazil. Univariate statistics was used as a method of analysis and the mean differences were calculated using paired T-Test groups. In a data analysis environment, statistically significant differences between the mean amplitudes were observed. Among the key findings was a considerable influence of aspects related to the brand on the cognitive processes that assign perceived value to certain products as well as the presence of anchoring bias and adjustments in the consumer decision making.
\end{abstract}

Keywords: Consumer behavior, pricing, decision making, anchoring, brand.

\section{Introdução}

A partir da Segunda Guerra Mundial percebe-se uma maior representatividade acadêmica de campos comportamentais de pesquisa, principalmente nas áreas relacionados ao consumo, o que pode ser resultado de maior profusão dos meios de comunicação e maior disponibilidade de produtos e nova caracterização da concorrência (Sousa, 2012).

Estudos relacionados ao comportamento do consumidor são oriundos da teoria econômica. De acordo com o principio microeconômico, todo o consumidor é dotado de uma renda limitada que por sua vez deve ser alocada de maneira a buscar a maximização de seu bem estar (Sousa, 2012). Neste aspecto, Baker (2000), ao contextualizar a essência do marketing também remonta a teoria econômica, ao apresentar o marketing como elemento intrínseco das relações de troca. 0 autor apresenta as relações entre oferta e demanda como fatores decisivos para a discussão relativa ao papel do marketing. Dado que os indivíduos têm necessidades ilimitadas, ou seja, não é possível prever com antecedência um limite para as necessidades, os recursos tornam-se escassos, pois, não existem bens em quantidade suficiente para atender às necessidades de todos na sociedade. 
Na atualidade é possível destacar o caráter interdisciplinar da teoria do comportamento do consumidor, envolvendo áreas como a psicologia, a economia, a sociologia, a antropologia, a administração e, a partir de meados da década de 1980, as neurociências. Nesse sentido, o estudo do comportamento do consumidor oferece contribuições para a ampliação do escopo de pesquisas de caráter social. Sendo que, para sua compreensão, faz-se mister compreender a relevância social dos atributos envolvidos na tomada de decisão dos atores envolvidos em um processo decisório.

Especificamente para o escopo deste trabalho optou-se por centrar as análises acerca da importância da marca na tomada de decisão dos consumidores. Notavelmente, nos dias atuais identifica-se uma expressiva manifestação, na qual marcas de produtos assumem posições de destaque (Zozzoli, 2012). Tal movimento é definido por Batey (2010) como a "Marca Pós-moderna". Segundo o autor, ao acrescentar "algo mais" (imagem, símbolos, percepções e sentimentos) a um produto físico, ele se torna uma marca, tendo como resultante deste processo um valor total superior ao da soma de suas partes. Sendo seu posicionamento mercadológico o que a define como pósmoderna (Batey, 2010).

Especialmente a partir das duas últimas décadas do século $\mathrm{XX}$, a sociedade atravessa um intenso e contínuo processo de modelização social, no qual tais ícones e símbolos são criteriosamente construídos, sofrendo influências das ações de marketing, comunicação, propaganda e programação visual (Zozzoli, 2012). Nesse ambiente, segundo Tavares (2008), a marca pós-moderna assume diferentes posicionamentos sociais, que por sua vez variam de indivíduo para indivíduo.

Torna-se possível inferir que o valor a ser atribuído a uma marca pode ser calculado a partir da resultante oriunda da convergência de diversas variáveis macro e micro ambientais. Nesse sentido, a marca possui um elevado grau de volatilidade, ou seja, a atribuição do valor de uma marca pode sofrer influência de fatores socioeconômicos e sociodemográficos, como sexo, faixa de renda e estado civil, por exemplo. Bem como de fatores psicológicos individuais, como, por exemplo, necessidade de pertencimento social, aprendizagem e atitude (Engel, Blackwell \& Miniard, 2000; Kotler, 2000; Sousa, 2012; Fernandes \& Correia, 2013; Brochado, Marques \& Mendes, 2015; Nascimento, Pereira, Sousa, Rezende \& Bueno, 2015).

Em complemento a esse posicionamento, Araújo e Loureiro (2014) ressaltam que tal processo torna-se ainda mais evidente quando é analisada a marca de luxo, uma vez que, segundo os autores, esta representa a melhor expressão das emoções e dos desejos humanos, dado seu poder de exemplificar a subjetividade do indivíduo, bem como de identificar a influência de aspectos culturais sob este.

Mediante ao exposto, interessa neste estudo, portanto, investigar questões relativas à diferença de valorização de produtos similares, porém de marcas distintas - em outros termos: é possível perceber influências estatisticamente consideráveis de vieses cognitivos na atribuição de preço a produtos similares, porém de marcas distintas?
Para responder tal questionamento, o presente estudo tem como objetivo geral identificar a existência diferenças estatisticamente significantes na precificação de produtos similares, porém de marcas distintas, tendo por base a atribuição de âncoras de decisão, a partir de uma marca notadamente conhecida pelo público pesquisado.

De forma específica pretende-se: a) analisar a importância da marca para os consumidores pesquisados; b) identificar se o fator renda exerce influência considerável no processo de precificação, c) identificar se existem diferenças de ancoragem nas percepções dos respondentes, baseadas no tipo do produto.

O artigo está dividido em cinco seções, incluindo esta introdução onde foram apresentados o tema do trabalho, problema e objetivos da pesquisa. Na segunda seção apresenta-se o referencial teórico que trata os seguintes temas: comportamento do consumidor, tomada de decisão em processos não declarativos, a marca pós-moderna e heurísticas, ancoragem e julgamento. Na terceira seção apresenta-se a metodologia, seguida da análise de dados e considerações finais.

\section{Referencial teórico}

Nesta seção apresenta-se o referencial teórico onde foram discutidos os seguintes temas: comportamento do consumidor, tomada de decisão em processos não declarativos, a marca pós-moderna e heurísticas.

\subsection{0 comportamento do consumidor}

Atualmente, no campo de estudo do marketing a área que mais fornece pesquisas de cunho acadêmico é a área que trabalha com o comportamento do consumidor. A teoria do comportamento do consumidor originou-se na teoria econômica, a partir do princípio microeconômico de que o consumidor é dotado de renda limitada, a qual deve se alocada entre bens e serviços de forma a buscar uma constante maximização de seu "bem estar" (Engel et al., 2000).

O estudo do comportamento do consumidor constitui um campo interdisciplinar de pesquisa e seu interesse pode ser associado no período que sucedeu a Segunda Guerra Mundial, especialmente pela superação da demanda reprimida, a inserção dos grandes centros de compras e a presença mais significativa da televisão como meio de entretenimento nas residências das pessoas (Engel et al., 2000). Atualmente recebe contribuições de diversas áreas do conhecimento como a ciência econômica (Sousa, 2012; Tversky \& Kahneman, 1974), a Psicologia (Bagozzi, 2002), Antropologia (Douglas \& Ishewood, 2009); Sociologia (Featherstone, 1997) e, atualmente, a Neurociência (Glimcher, 2012; Plassmann, 2012; Sousa, 2012; Berčík, Horská, Wang \& Chen, 2015; Dimpfel, 2015; Schwarzkopf, 2015).

De acordo com Engel et al. (2000), os primeiros estudos nessa área concentravam seus esforços na tentativa de tornar os consumidores leais e assim adquirir diferenciais competitivos para as organizações. Os autores ainda complementam que para tanto, o entendimento de seu perfil, suas motivações e expectativas acerca de um determinado produto seria crucial. A partir da década de 
1980 as pesquisas na área passaram a incluir aspectos como: simbolismos, experiências passadas e impactos das religiões e emoções (Sheth, Gardner \& Garret, 1998). Em complemento, Gonçalves, Menezes e Marques (2015) salientam que o comportamento do consumidor pode ser compreendido como a resultante de um processo de convergência de diversos fatores, dentre os quais se destacam os sociais, psicológicos, pessoais e culturais, sendo este último que, segundo Fernandes e Correia (2013), exerce maior influência no comportamento individual. Atualmente este campo de estudo se tornou mais amplo, especialmente a partir das contribuições recebidas da Psicologia e da Neurociência, que estudam aspectos não declarativos na tomada de decisão (Dias, 2010).

Segundo Kotler (2000), o comportamento do consumidor está diretamente relacionado ao processo de troca. Solomon (2002) acrescenta que, embora o processo de troca em si seja importante, deve-se considerar uma visão de maior abrangência do processo, a qual influências de antes, durante e depois do ato de consumo devem ser incluídas. Para Engel et al. (2000), diversas áreas atuam juntas em um sistema de base psicossocial específico, o qual permeia o processo de tomada de decisão do consumidor.

De acordo com Pinto e Lara (2010) considerar o consumidor como um ser racional, que busca tomar as melhores decisões nas relações de troca, implica negligenciar aspectos importantes relacionados ao simbolismo presente em tais dinâmicas. Para os autores, o ato de comprar não deve ser dissociado se sua representação como forma de socialização, estando o consumo imbuído de sentimentos diversos como, prazer, emoção, alegria, raiva, frustação, reconhecimento e tédio.

\subsection{Tomada de decisão em processos não-declarativos}

A partir do início da década de 1990 percebe-se um aumento do interesse, tanto acadêmico quanto social, no processo de tomada de decisão. Notavelmente, tal processo propagou-se além das fronteiras das ciências econômicas ao ponto de ser considerado interdisciplinar (Sousa, 2012; Sousa, Lara, Costa, Gonçalves, Bressan \& Dias, 2013).

Na tentativa de compreensão desse processo, ciências como a antropologia, a psiquiatria, a psicologia, a sociologia, a filosofia, a neurociências, dentre outras, fornecem embasamento teórico e empírico. Nesse sentido, vale destacar que a compreensão dos fatores que influenciam a tomada de decisão torna-se insumo valioso para um entendimento mais efetivo do comportamento do consumidor. Falar em tomada de decisão implica em um entendimento fisiológico do sistema cognitivo. Sendo assim, torna-se necessária uma compreensão dos processos bioquímicos que permeiam o funcionamento do cérebro.

O cérebro pode ser representado por uma ampla e complexa rede de ligações neuronais na qual, por um ponto de vista biocomputacional, utiliza-se de inputs de caráter verbal e não verbal, conscientes ou inconscientes, no processo de tomada de decisão (Dias, 2010). Ele pode ser entendido como um sistema bayesiano, ou seja, trata-se de um sistema visto como uma máquina de probabilidades em constante realização de previsões acerca do mundo, simultaneamente atualiza com base em seus sentidos (Tokuno \& Tanaka, 2011).

Em consonância a este pensamento entende-se por tomada de decisão a resultante do processo pelo qual decisões rearranjam representações de objetos, de forma concreta ou hipotética, diminuindo de forma transitória a entropia do sistema cognitivo (Dias, 2010). Ainda de acordo com o autor, este processo gera uma vetoralidade no sistema cognitivo que, na maioria das vezes, se dá a partir de um transitório aumento entrópico do organismo como um todo, sendo sequentemente determinado pelo empenho intencional para a realização em ato da decisão.

Nesse sentido, torna-se possível inferir que a tomada de decisão é uma ação pela qual se tem a diminuição da entropia no sistema cognitivo e particularização de um processo genérico, além de envolver mecanismos de valorização e recompensa (Dias, 2010; Doya \& Shadlen, 2012; Gleichgerrcht, Ibanez, Manes, Roca \& Torralva, 2010).

Esse complexo sistema ainda possui o que de acordo com Dias (2010) pode ser chamado de inconsciente decisório, parte da mente na qual o ser humano não possui um controle direto. 0 autor ainda completa que o acesso a esta camada da mente revela a mecânica da redução entrópica pela força da escolha, sendo então elas responsáveis pela tomada de decisão propriamente dita.

\subsection{A marca pós-moderna}

A definição clássica de marca foi desenvolvida nos anos 1960 pela American Marketing Association (AMA), a qual enfatizava a figura visual como uma forma de diferenciar uma marca das outras, "um nome, um termo, um sinal, um símbolo, um desenho ou uma combinação entre eles, que tencione identificar os bens e serviços de um vendedor ou de um grupo de vendedores e diferenciá-los dos competidores" (Chernatony \& Riley, 1997, p. 48). Segundo Batey (2010), ao acrescentar "algo mais" (imagem, símbolos, percepções e sentimentos) a um produto físico, ele se torna uma marca. Este processo produz um valor total superior ao da soma de suas partes. Segundo o autor, uma marca pode representar um produto específico bem como a composição de vários produtos de diversas categorias diferentes.

Tavares (2008) destaca a importância da antropologia do consumo no processo de compreensão da marca pós-moderna, uma vez que o campo busca compreender o significado simbólico do ato de consumir e a marca pós-moderna, por sua vez, se estrutura em bases simbólicas. Para o autor, o consumo deve ser visto como um sistema de significação para suprir uma necessidade simbólica. Ou seja, o papel do consumo deve ser entendido como forma de "construir significados pessoais e coletivos, estabelecer, evidenciar e estabilizar categorias culturais como sistemas de classificação no mundo que nos cerca" (Tavares, 2008, p. 275).

Destaca-se que a criação de uma marca se estrutura, principalmente, a partir do resultado da leitura do consumidor sobre atividades exercidas sobre ele (estímulo, input) (Nascimento et al., 2015). O consumidor escolhe uma marca a partir do que ela significa para ele, ou seja, para o consumidor a marca é uma série de associações, expectativas e percepções que existem em sua cabeça, 
sendo elas criadas, mantidas ou até mesmo incrementadas a partir de experiências e encontros que o consumidor tem com ela (Batey, 2010; Foxal, 2012; Brochado et al., 2015).

De acordo com Semprini (2010), a marca é estruturada a partir de três dimensões fundamentais, a natureza semiótica, a natureza racional e a natureza evolutiva. Para o autor, a natureza semiótica está relacionada ao significado e ao sentido, ou seja, a partir dessa natureza torna-se possível construir e veicular significados a marca. Pode-se dizer que esta é a dimensão mais característica da marca uma vez que o espaço social em sua totalidade apresenta grandes preocupações de caráter semiótico (busca de sentido, valores imateriais, midiatização generalizada).

Por sua vez, a natureza relacional da marca decorre em parte de sua natureza semiótica, e em parte de seus mecanismos de funcionamento. No interior desta natureza é possível identificar dois aspectos principais de sua estrutura, inicialmente uma dimensão intersubjetiva, segundo a qual a marca é uma resultante, sempre de caráter provisório, de um contínuo processo de trocas e de negociações que implica em diversos papeis de um número grande de protagonistas (Semprini, 2010). A segunda, uma dimensão contratual, pela qual:

A lógica de troca que está sempre implícita em uma lógica de marca é alimentada por uma promessa e solicita os destinatários dispostos a reconhecer um valor (funcional, simbólico) a de um bem ou serviço em troca de dinheiro. (...) Para que a noção de contrato adquira todo o seu sentido, é preciso que as trocas sejam repetidas no tempo. A dimensão contratual é então muito importante, pois ela inscreve a marca na duração e a obriga a uma regularidade e a uma continuidade temporal (Semprini, 2010, p. 104).

Por fim, o autor destaca a natureza evolutiva, sendo ela oriunda de um caráter dinâmico mutável da marca, ou seja, evolução contínua. Nesta, considera-se a marca como uma entidade viva, que reage às mudanças que lhe são impostas, principalmente de ambiente. Vale destacar que este caráter dinâmico originase das modalidades relacionais de sua geração.

\subsubsection{A percepção da marca pós-moderna}

A percepção de uma marca reflete sua realidade. Nesse sentido, Lindstrom (2007) discute a importância sensorial na constituição da mesma. Para o autor, os cinco sentidos humanos devem ser utilizados em um processo de branding, ou seja, a comunicação com o consumidor deve transcender os métodos convencionais. Marcas inconfundíveis necessitam de um diferencial, precisam ser capazes de transmitir e entregar um valor sensorial e emocional aos seus consumidores.

O branding sensorial atribui quatro dimensões importantes à marca, sendo inicialmente um compromisso emocional do consumidor, em segundo uma equivalência otimizada entre percepção e realidade, seguida da criação de uma plataforma de marca única para diferentes linhas de produtos e por fim, uma marca diferenciada, inigualável e única (Lindstrom, 2007).

Torna-se válido destacar que o cérebro humano, órgão responsável pelos sentidos, possui uma série de interligações neuronais, não sendo possível a separação sensorial em um indivíduo comum, sem nenhuma patologia ou deficiência sensorial (Sousa, 2012; Sousa et al. 2013). Nesse sentido, a exploração sensorial de uma marca deve ser estruturada de forma a compreender todos os sentidos humanos, caso contrário, possivelmente não serão alcançados os resultados almejados (Lindstrom, 2007).

\subsection{Heurísticas, ancoragem e ajustamento}

Considerando-se as limitações cerebrais humanas quanto à capacidade de processamento de informações, o cérebro cria atalhos mentais, que na literatura são chamadas heurísticas cognitivas, sendo elas utilizadas no processo de tomada de decisão (Bezerra, 2011). Segundo Tonetto, Kalil, Melo, Schneider \& Stein (2006) as heurísticas também podem ser vistas como regras de influência que os sujeitos utilizam para a formação de julgamento em processos de tomada de decisão sob condições de incertezas.

Por se tratarem de atalhos que reduzem a complexidade e o tempo necessário para a realização de algumas tarefas, as heurísticas podem levar a erros graves e sistemáticos (Tversky \& Kahneman, 1974).

Nesse sentido, para Baker e Nofsinger (2002), na tentativa de diminuição da complexidade do processo decisório de informações que exigem uma maior análise do sujeito, o intelecto cria um filtro que bloqueia algumas dessas informações consideradas desnecessárias e utiliza algumas heurísticas no processo. Contudo, estes atalhos podem fazer com que o cérebro crie respostas antes do processamento integral de informações disponíveis, chamados de simplificação heurística, que podem levar o sujeito a vieses psicológicos e a erros de avaliação (Bezerra, 2011).

Uma das heurísticas mais comumente utilizadas, a ancoragem, é o processo pelo qual a mente do decisor dá preferência às primeiras informações que lhe são fornecidas (Bezerra, 2011).

Segundo Tversky e Kahneman (1974), em processos de julgamentos sob elevado grau de incertezas, quando o indivíduo deve realizar estimativas ou até mesmo tomar decisões sobre alguma quantia, ele tende a criar uma estrutura psicológica a fim de ajustar sua resposta tendo como base um valor inicial disponível, a chamada âncora. A este atalho cognitivo é dado o nome de heurística de "ancoragem e ajustamento", ou seja, trata-se de um processo pelo qual a âncora proposta inicialmente exerce influência sobre a decisão final do indivíduo.

Segundo Bezerra (2011, p. 35) "a heurística da ancoragem pode ser percebida quando um valor inicial é prontamente referencial na predição de estimativas numéricas". Nesse sentido, Tversky e Kahneman (1974) afirmam que muitas decisões, principalmente aquelas tomadas em ambientes de incertezas e tensões, são tomadas com base em uma âncora, que por sua vez é ajustada e levada à resposta final. Contudo, destacam os autores que este ajustamento pode ser insuficiente e, consequentemente, enviesar o resultado final.

\section{Metodologia}

0 presente estudo possui abordagem quantitativa, ou seja, parte do princípio que todos os dados são quantificáveis. A partir da coleta todos os dados são tabulados e analisados a luz da estatística (Collis \& Hussey, 2005). 
Quanto aos fins trata-se de um estudo cuja natureza é empírico-descritiva, ou seja, tem como objetivo descrever situações mercadológicas a partir de dados primários (Collis \& Hussey, 2005). Segundo Samara e Barros (2002), as pesquisas descritivas são utilizadas, geralmente, quando se tem um propósito de criar uma descrição de características de grupos.

Quanto aos meios, a presente pesquisa consiste de um estudo de campo do tipo survey on-line (Cooper \& Schindler, 2003). A coleta de dados deu-se via questionário estruturado, validado por Sousa (2012), e adaptado aos propósitos deste trabalho.
0 instrumento continha 28 questões, sendo: na primeira etapa da pesquisa, os participantes visualizavam uma imagem de um produto de luxo com o seu respectivo valor de mercado e em seguida visualizavam uma imagem de uma réplica do mesmo produto, para a qual foi dado um nome fictício. Os entrevistados deveriam atribuir um valor financeiro a réplica, que ele julgasse justo. Na segunda etapa da pesquisa, os participantes visualizavam uma imagem de uma réplica de um produto de luxo, com o mesmo nome fictício, para o qual foi atribuído um valor de mercado. $\mathrm{Na}$ sequência, era apresentado o produto original, o qual eles deveriam fazer a precificação, conforme julgassem justo. No quadro 1 é apresentada a relação de imagens, marcas e valores dos produtos utilizados na pesquisa:

\section{Quadro 1 - Relação das marcas trabalhadas}

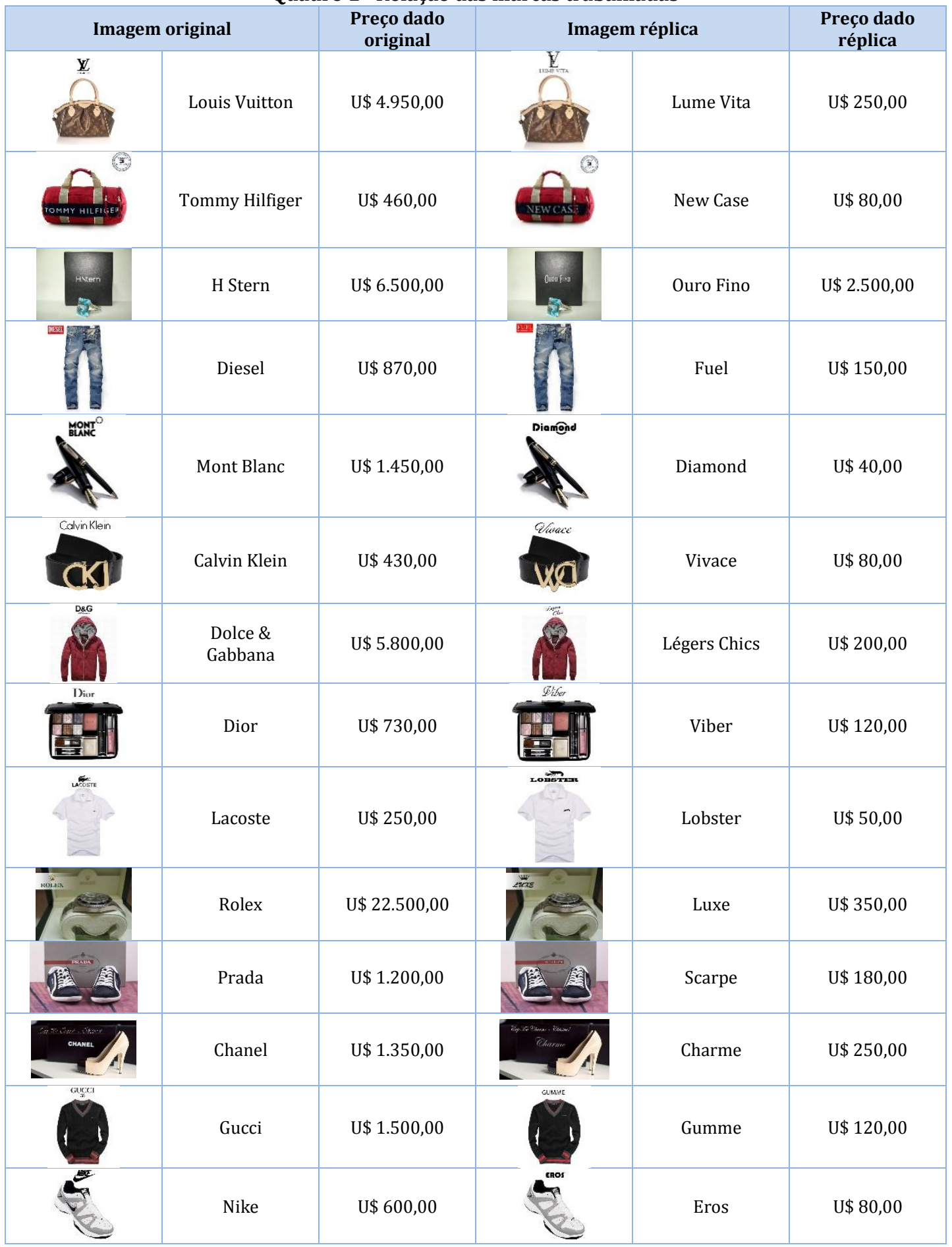

Fonte: Dados da pesquisa. 
A pesquisa ficou disponível no período de 15 de outubro de 2013 a 15 de novembro de 2013. Um total de 183 pessoas iniciou o preenchimento do questionário, sendo que, deste número, 111 pessoas o finalizaram. Todos os respondentes residem no estado de Minas Gerais, que é o segundo mais populoso do Brasil. $\mathrm{Na}$ análise de dados optou-se por realizar a diferença entre médias das diferenças de preço atribuídos aos produtos, utilizando para tanto T-Test de grupos pareados, realizados por meio do programa estatístico SPSS (Statistical Package for the Social Sciences).

\section{Análise de dados}

\subsection{Caracterização dos respondentes}

A amostra foi constituída por 111 respondentes, dos quais $63,1 \%$ são do sexo feminino e $36,9 \%$ do sexo masculino. Quanto à faixa etária, $5,4 \%$ dos respondentes possuem idade entre 16 e 20 anos de idade; $53,2 \%$ entre 21 e 30 anos; $29,7 \%$ entre 31 e 45 anos; $10,8 \%$ entre 46 e 60 anos e $0,9 \%$ tem idade acima de 65 anos.

Em relação à distribuição por renda, 2,7\% dos respondentes afirmaram não possuir renda na data da pesquisa; $27,0 \%$ declararam possuir renda de até U\$608,00; 36,9\% entre $\mathrm{U} \$ 609,00$ a U\$1520,00; 23,4\% entre U\$1521,00 e $\mathrm{U} \$ 3045,00 ; 3,6 \%$ entre U\$3045,00 e U\$4560,00; 5,5\% entre
U\$4560,00 e U\$6080,00 e 0,9\% possuem renda superior a $\mathrm{R} \$ 6081,00$.

A maior parte da amostra $(64,9 \%)$ foi constituída por solteiros; $24,3 \%$ são casados ou possuem união estável; $0,9 \%$ são viúvos; $8,1 \%$ são divorciados ou desquitados e $1,8 \%$ marcaram o estado civil outros.

\subsection{Demonstração de resultados}

Com base na heurística da ancoragem, esperava-se que a diferença observada na primeira pergunta (preço de mercado informado de um produto original menos o valor percebido de uma réplica) fosse superior a diferença observada na segunda pergunta (valor de mercado informado de uma réplica menos o valor percebido do original). Tal fato ocorreria devido à maior exposição de consumidores a informações de preço das réplicas em comparação com os originais, o que provavelmente os levaria a oferecer respostas sobre o preço de réplicas mais condizentes com a realidade independentemente da informação sugerida inicialmente.

Para testar tal hipótese, realizou-se testes de diferenças entre as médias das marcas, utilizando para tanto T-Test de grupos pareados. Os resultados dos testes estatisticamente significantes são apresentados na tabela 1.

Tabela 1 - Diferenças entre médias

\begin{tabular}{|c|c|c|c|c|}
\hline Marca & Diferenças & Média & Desvio Padrão & Erro Padrão \\
\hline \multirow{2}{*}{ Louis Vuitton } & Ori./Rep. & $\mathrm{U} \$ 1.854,15$ & U\$ 648,27 & $\mathrm{U} \$ 61,53$ \\
\hline & Rep./Ori. & U\$ 640,51 & U\$ $1.369,43$ & U\$ 129,98 \\
\hline \multirow{2}{*}{ H Stern } & Ori./Rep. & U\$ 1.969,51 & U\$ 967,88 & U\$ 91,87 \\
\hline & Rep./Ori. & U\$ $1.102,09$ & U\$ $1.841,04$ & U\$ 174,74 \\
\hline \multirow{2}{*}{ Mont Blanc } & Ori./Rep. & U\$ 500,45 & U\$2 206,72 & U\$ 19,62 \\
\hline & Rep./Ori. & U\$ 211,19 & U\$ 553,61 & U\$ 52,55 \\
\hline \multirow{2}{*}{ Dolce Gabbana } & Ori./Rep. & $\mathrm{U} \$ 2.170,61$ & U\$ 793,70 & U\$ 75,33 \\
\hline & Rep./Ori. & U\$ 307,36 & U\$ $1.179,40$ & U\$ 111,94 \\
\hline \multirow{2}{*}{ Rolex } & Ori./Rep. & U\$ 8.356,16 & U\$ $2.900,09$ & U\$ 275,26 \\
\hline & Rep./Ori. & $\mathrm{U} \$ 2.233,91$ & U\$ 8.711,67 & $\mathrm{U} \$ 826,87$ \\
\hline \multirow{2}{*}{ Prada } & Ori./Rep. & $\mathrm{U} \$ 410,52$ & U\$ 144,12 & $\mathrm{U} \$ 13,68$ \\
\hline & Rep./Ori. & U\$ 157,82 & U\$ 465,86 & U\$ 44,22 \\
\hline \multirow{2}{*}{ Chanel } & Ori./Rep. & $\mathrm{U} \$ 454,73$ & U\$ 164,75 & U\$ 15,64 \\
\hline & Rep./Ori. & U\$ 204,70 & U\$ 469,91 & $\mathrm{U} \$ 44,60$ \\
\hline \multirow{2}{*}{ Gucci } & Ori./Rep. & U\$ 544,68 & U\$ 181,56 & $\mathrm{U} \$ 17,23$ \\
\hline & Rep./Ori. & U\$ 138,84 & U\$ 419,40 & U\$ 39,81 \\
\hline \multirow{2}{*}{ Nike } & Ori./Rep. & U\$ 179,62 & U\$ 70,96 & $\mathrm{U} \$ 6,73$ \\
\hline & Rep./Ori. & U\$94,06 & U\$ 106,67 & U\$ 10,12 \\
\hline
\end{tabular}

De acordo com os resultados dos testes, em uma primeira análise, percebeu-se que os nove produtos apresentados apontaram uma amplitude média da diferença original/réplica (Dif. Ori/Rep) consideravelmente superior a amplitude média da diferença réplica/original. Ou seja, o intervalo gerado entre os valores atribuídos na primeira parte da pesquisa são maiores que os intervalos de valores gerados na segunda etapa.

Em uma segunda análise, percebe-se que em todos os produtos, o desvio padrão se mostra superior na diferença réplica/original (Dif. Rep/Ori). Tal fato leva a inferir que os dados encontrados nos resultados individuais de precificação dos produtos, nesta fase da pesquisa, se mostram mais dispersos, ou seja, há uma maior variação dos valores atribuídos aos produtos originais.

Em consonância aos dados apresentados, identificou-se uma maior incidência da heurística da ancoragem e ajustamento na segunda etapa da pesquisa, precificação réplica/original.

A presença da heurística pode ser explicada inicialmente pelo fato de que, a maioria dos respondentes se encontrava na faixa de renda entre U\$609,00 a U\$1520,00 (36,9\% dos entrevistados), sendo assim, os valores atribuídos aos produtos originais tendem a sofrer maior ancoragem e ajustamento, uma vez que os valores referentes aos 
produtos réplicas são mais comuns ao dia a dia da maior parte da amostra.

Comprovou-se então parte da hipótese levantada que a diferença observada na primeira pergunta (preço de mercado informado de um produto original menos o valor percebido de uma réplica) fosse superior a diferença observada na segunda pergunta (valor de mercado informado de uma réplica menos o valor percebido do original. A tabela 2 apresenta as diferenças entre as médias.

Tabela 2 - Diferenças entre médias das marcas

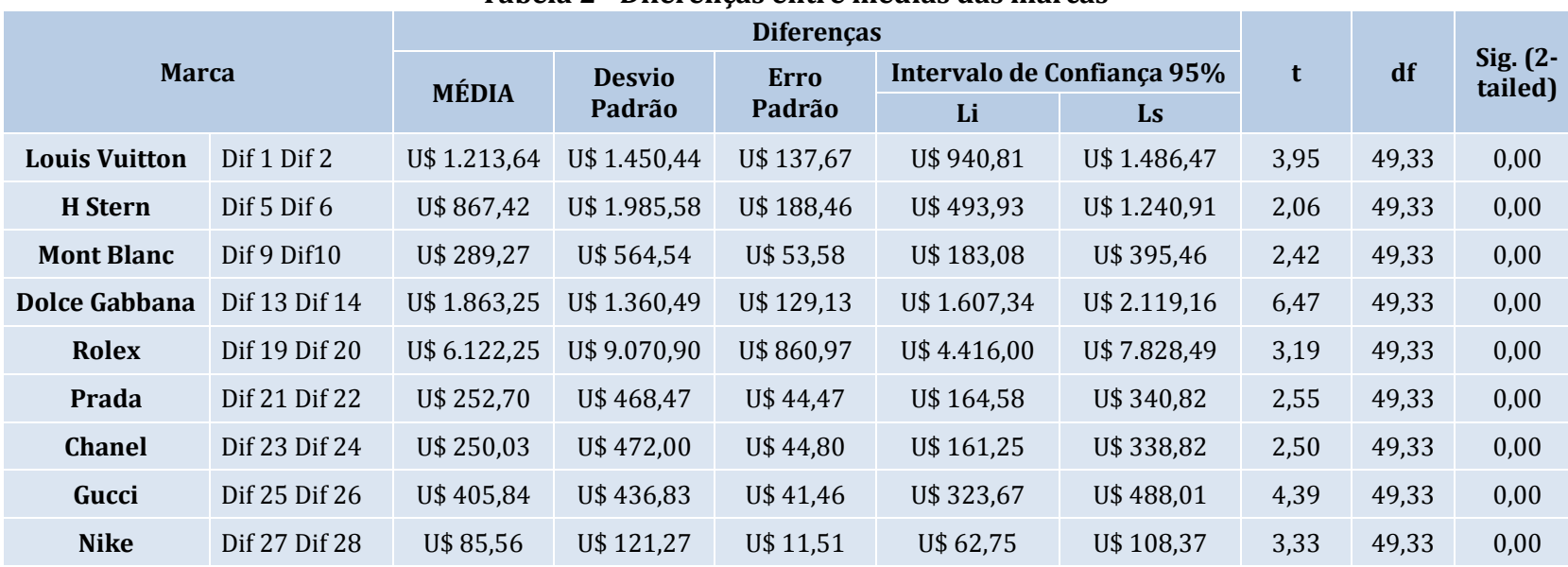

Fonte: Dados da pesquisa.

Tendo como ponto de partida a análise da diferença das diferenças de médias, observou-se que para os 14 produtos analisados, 9 resultaram em médias com diferenças estatisticamente significante $(\mathrm{IC}=95 \%)$, para os quais a média das diferenças da primeira pergunta apareceu superior a média das diferenças da segunda pergunta, comprovando assim a segunda parte da hipótese conforme o esperado.

A análise também possibilitou quantificar a importância da marca no processo de atribuição de valor a determinado produto. Tendo como base, o fato de que a diferença das diferenças das médias em $64,3 \%$ dos casos se mostrou estatisticamente significante.

Na seção seguinte são apresentadas as considerações finais e sugestões para estudos futuros.

\section{Considerações finais}

Atualmente, a sociedade, tendo como foco o consumidor, se encontra em meio a uma expressiva e contínua revolução promovida por modificações mercadológica, sendo várias destas pautadas em um ambiente de maior disponibilidade de produto, via intensificação da globalização e meios de comunicação (Batey, 2010; Tavares, 2008). Nesse ambiente, fatores intangíveis buscam agregar valor ao produto, aumentando assim sua competitividade. Esse movimento é chamado de "Marca Pós Moderna".

Para a compreensão da relevância da marca pós-moderna no comportamento do consumidor, inicialmente deve-se ter em vista sua natureza evolutiva, sendo ela oriunda de um caráter dinâmico mutável da marca. Nesse sentido, o problema de pesquisa se propôs a investigar a existência e a influência de vieses cognitivos no processo de tomada de decisão do consumidor (para o caso específico de precificação de produtos).

No presente artigo, a partir de uma análise dos valores atribuídos pelos respondentes da survey online, identificouse inicialmente uma considerável influência de aspectos relacionados à marca no processo de atribuição de 'preço justo' a produtos "Originais de Luxo" e "Réplicas" do mesmo, assim como identificado por Semprini (2010).

Inicialmente identificou-se uma maior amplitude de diferenças de preços para os produtos Réplica quando precificados, na primeira etapa da pesquisa, tendo por base produtos originais. A investigação de tal fato conduziu a tentativa de quantificar a importância que a maioria dos consumidores atribui à marca, que muitas vezes é compreendida como sinal de qualidade.

Em um segundo momento percebeu-se a influência da heurística da ancoragem e ajustamento no processo de precificação. Tal viés pode ser observado pelo resultado da diferença de médias da primeira pergunta (preço de mercado informado de um produto original menos o valor percebido de uma réplica), que se mostrou superior a diferença observada na segunda pergunta (valor de mercado informado de uma réplica menos o valor percebido do original).

A razão da ocorrência do viés pode ser atribuída ao fato de uma maior exposição de consumidores a informações de preço das réplicas em comparação com os originais, fato este que leva a confirmar a hipótese de que a faixa de renda na qual o sujeito se encontra exerce influência considerável no processo de ancoragem. A ocorrência de tal fato, provavelmente, levou a oferecer respostas sobre o preço de réplicas mais condizentes com sua realidade econômica, independentemente da informação sugerida inicialmente.

Nesse sentido, vale ressaltar que as imagens utilizadas para a elaboração do presente estudo eram as mesmas, a única alteração era no nome atribuído ao produto, ora o nome da marca original do produto, ora um nome de marca fictícia. Sendo assim, a partir dos dados apresentados, identificouse que a marca é um fator importante nos processos cognitivos de percepção e atribuição de valor a determinados produtos.

0 presente estudo investigou a presença e influência de um único viés, a ancoragem e ajustamento, na precificação de produtos. Sendo assim, sugere-se o desenvolvimento de futuros estudos que contemplem a influência de outros vieses cognitivos no processo de tomada de decisão do consumidor. Sugere-se ainda, investigações de caráter qualitativo, para compreender o porquê cinco dos produtos analisados (bolsa Tommy Hilfiger, calça Diesel, cinto Calvin Klein, maquiagens Dior e blusa Lacoste) não possuíram resultados estatisticamente significantes. 


\section{Referências}

Araújo, C. M. B. D., \& Loureiro, S. M. C. (2014). 0 efeito da norma subjetiva, percepção de controlo, atitude e experiência passada na intenção de compra de marcas de roupa de luxo no Brasil. Tourism \& Management Studies, 10(Special Issue), 103-110.

Bagozzi, R. P., Gurhan-Canli, Z. \& Priester, J. R. (2002). The social psychology of consumer behaviour. Buckingham: Open University Press.

Baker, H. K. \& Nofsinger, J. R. (2002). Psychological biases of investors. Financial Service Review, 11(2), 97-116.

Baker, M. J. (200). Marketing: philosophy or function? London: Business Press.

Batey, M. (2010). O significado da marca: como as pessoas ganham a vida na mente dos consumidores. Rio de Janeiro: Best business.

Berčík, J., Horská, E., Wang, W. Y., \& Chen, Y. C. (2015). How can food retailing benefit from neuromarketing research: a case of various parameters of store illumination and consumer response. In 143rd Joint EAAE/AAEA Seminar, March 25-27, 2015, Naples, Italy (No. 202714). European Association of Agricultural Economists.

Bezerra, J. M. F. (2011). A heurística da ancoragem e seus efeitos em estimativas de preço: um estudo com produtos da cesta básica. Dissertação de mestrado, Universidade Potiguar, Potiguar, Natal, Brasil.

Brochado, A. O., Marques, S. H., \& Mendes, P. (2015). Psychographic determinants of private-label adoption: a feasibility study in the Portuguese yogurt market. Tourism \& Management Studies, 11(1), 136-145.

Chernatony, L. \& Riley, D. O. F. (1997) Brand consultants perspectives on the concept of the brand. Marketing and Research Today, 25(1), 45-52.

Collis, J. \& Hussey, R. (2005). Pesquisa em administração: um guia prático para alunos de graduação e pós-graduação. (2a ed.). Porto Alegre: Bookman.

Cooper, D. R. \& Schindler, P. S. (2003). Métodos de pesquisa em administração. (7a ed.). Porto Alegre: Bookman.

Dias, A. M. (2010). Processos não declarativos em tomadas de decisão: modelos e experimentos. Tese de doutorado, Universidade de São Paulo, São Paulo, SP, Brasil.

Dias, A. M. (2012). Das 'neurociências aplicadas ao marketing' ao 'neuromarketing integrativo'. Ciências \& Cognição, 17(1), 178-189.

Dimpfel, W. (2015). Neuromarketing: Neurocode-Tracking in Combination with Eye-Tracking for Quantitative Objective Assessment of TV Commercials. Journal of Behavioral and Brain Science, 5(04), 137.

Douglas, M. \& Ishewood, Baron. (2009). O mundo dos bens: para uma antropologia do consumo. Rio de Janeiro: Editora UFRJ.

Doya, K. \& Shadlen, M. (2012). Decision making. Neurobiology, 22(1), 911-913.

Engel, J. F., Blackwell, R. D. \& Miniard, P. W. (2000). Comportamento do consumidor. (8a ed.). Rio de Janeiro: Livros Técnicos e Científicos Editora S.A.

Featherstone, M. (1997). A autonomização da esfera cultural. São Paulo: Studio Nobel.

Fernandes, P. O., \& Correia, L. F. (2013). Atitudes do consumidor em relação às práticas do marketing em Portugal. Tourism \& Management Studies, 9(2), 86-92.

Gleichgerrcht, E., Ibanez, A., Manes, F., Roca, M. \& Torralva, T. (2010). Decision-making cognition in neurodegenerative diseases. Nature Reviews Neurology, 6(2), 611-621.

Glimcher, P. W. \& Fehr, E. (2012). Decision making and the brain. London: Elsevier Inc.

Gonçalves, M. F., Menezes, J., \& Marques, C. (2015). Grocery consumer relational perceptions in green consumption context. Tourism \& Management Studies, 11(1), 160-163.

Kotler, P. (2000). Administração de marketing: a edição do novo milênio. (10a ed.). São Paulo: Prentice Hall.

Lindstrom, M. (2007). Brandsense: a marca multissensorial. Porto Alegre: Bookman.

Nascimento, L. M. S., Pereira, J. R., Sousa, C. V., Rezende, L. B. O. \& Bueno, N. X. (2015). Cópia não! Réplica. Analisando o consumo além das fronteiras utilitárias. In: International Conference on Innovation and Entrepreneurship in Marketing and Consumer Behaviour, Aveiro, Portugal.

Pinto, M. R. \& Lara, J. E. (2010). 0 marketing de relacionamento no mercado consumidor em uma perspectiva experiencial e simbólica: buscando novas proposições para pesquisas. Anais do Encontro de Marketing da Associação Nacional de Pós-Graduação e Pesquisa em Administração, Florianópolis, SC, Brasil.

Plassmann, H., Ramsøy, T. Z., \& Milosavljevic, M. (2012). Branding the brain: a critical review and outlook. Journal of Consumer Psychology, 22(1), 18-36.

Samara, B.S. \& Barros, J. C. (2002). Pesquisa de marketing: conceitos e metodologia. (3a ed.). São Paulo: Prentice Hall.

Schwarzkopf, S. (2015). Measurement devices and the psychophysiology of consumer behaviour: A posthuman genealogy of neuromarketing. BioSocieties.

Semprini, A. (2010). A marca pós-moderna: poder e fragilidade da marca na sociedade contemporânea. (2a ed.). São Paulo: Estação das Letras e Cores.

Sheth, J. N., Gardner, D. M. \& Garrett, D. E. (1988). Marketing theory: evolution and evaluation. Chichester: Jonh Wiley \& Sons.

Solomon, M. R. (2002). Comportamento do consumidor: comprando, possuindo e sendo. (5a ed.). Porto Alegre: Bookman.

Sousa, C. V. (2012). Neurociências e marketing: explorando as fronteiras diádicas e integrando metodologias para a compreensão do comportamento do consumidor. Tese de doutorado, Universidade Federal de Minas Gerais, Belo Horizonte.

Sousa, C. V., Lara, J. E., Costa, E. C., Gonçalves, C. A., Bressan, R. A. \& Dias, A. M. (2013). O que é possível perceber além do declarado? A utilização do neuromarketing como estratégia de pesquisa em marketing. Anais do Encontro Nacional da Associação Nacional de Pós-Graduação e Pesquisa em Administração, Rio de Janeiro, RJ, Brasil, 37.

Tavares, M. C. (2008). Gestão de marcas: construindo marcas de valor. São Paulo: Harbra.

Tokuno, H., Tanaka, I. (2011). Decision making and risk attitude of the common marmoset in a gambling task. Neuroscience Research, 13(3), 11-17.

Tonetto, L. M., Kalil, L. L., Melo, W. V., Schneider, D. G. \& Stein, L. M. (2006). O papel das heurísticas no julgamento e na tomada de decisão sob incerteza. Estudos de Psicologia, 13 (2), 181-189.

Tversky, A. \& Kahneman, D. (1974). Judgment under uncertainty: heuristics and biases. Science, 185(4157), 1124-1131.

Zozzoli, J. C. J. (2012). A percepção da marca e do título de estabelecimento comercial: resultado de uma pesquisa qualitativa. Anais do Congresso Brasileiro de Ciências da Comunicação, Campo Grande, SP, Brasil, 24.

Submetido: 14.05 .2014

Aceite: 12.10.2014 